\title{
Explicit Solutions for the Solomon-Wilson-Alexiades's Mushy Zone Model with Convective or Heat Flux Boundary Conditions
}

\author{
Domingo A. Tarzia ${ }^{1,2}$ \\ ${ }^{1}$ Departamento de Matemática, FCE, Universidad Austral, Paraguay 1950, S2000FZF Rosario, Argentina \\ ${ }^{2}$ CONICET, Argentina \\ Correspondence should be addressed to Domingo A. Tarzia; dtarzia@austral.edu.ar
}

Received 14 June 2015; Revised 22 October 2015; Accepted 29 October 2015

Academic Editor: Mustafa Inc

Copyright (c) 2015 Domingo A. Tarzia. This is an open access article distributed under the Creative Commons Attribution License, which permits unrestricted use, distribution, and reproduction in any medium, provided the original work is properly cited.

\begin{abstract}
We complete the Solomon-Wilson-Alexiades's mushy zone model (Solomon, 1982) for the one-phase Lamé-Clapeyron-Stefan problem by obtaining explicit solutions when a convective or heat flux boundary condition is imposed on the fixed face for a semiinfinite material. We also obtain the necessary and sufficient condition on data in order to get the explicit solutions for both cases which is new with respect to the original model. Moreover, when these conditions are satisfied, the two phase-change problems are equivalent to the same problem with a temperature boundary condition on the fixed face and therefore an inequality for the coefficient which characterized one of the two free interfaces of the model is also obtained.
\end{abstract}

\section{Introduction}

Heat transfer problems with a phase-change such as melting and freezing have been studied in the last century due to their wide scientific and technological applications [1-9]. A review of a long bibliography on moving and free boundary problems for phase-change materials (PCM) for the heat equation is shown in [10]. The importance of obtaining explicit solutions to some free boundary problems was given in the works [11-26].

We consider a semi-infinite material, with constant thermal coefficients, that is initially assumed to be liquid at its melting temperature which is assumed to be equal to $0^{\circ} \mathrm{C}$. At time $t=0$, a heat flux or a convective boundary condition is imposed at the fixed face $x=0$, and a solidification process begins where three regions can be distinguished $[27,28]$ :

(H1) liquid region at the temperature $0^{\circ} \mathrm{C}$, in $x>r(t), t>$ 0 ;

(H2) solid region at the temperature $T(x, t)<0$, in $0<x<$ $s(t), t>0$ (with $s(t)<r(t)$ );

(H3) mushy region at the temperature $T(x, t)=0$, in $s(t) \leq$ $x \leq r(t), t>0$. The mushy region is considered isothermal and we make the following assumptions on its structure:

(H3i) the material contains a fixed portion $\varepsilon \mathcal{\ell}$ (with $0<\varepsilon<1$ ) of the total latent heat $\ell$ (see condition (3) in below);

(H3ii) the width of the mushy region is inversely proportional to the gradient of temperature (see condition (4) below).

Following the methodology given in [27-29] and the recent one in [30], we consider a convective boundary condition in Sections 2 to 4 and a heat flux condition in Sections 5 and 6 at the fixed face $x=0$, respectively. In both cases, we obtain explicit solutions for the temperature and the two free boundaries which define the mushy region. We also obtain, for both cases, the necessary and sufficient condition on data in order to get these explicit solutions given in Sections 2 and 5, respectively, which is new with respect to the original model when a temperature boundary condition at the face $x=0$ was imposed. Moreover, these two problems are equivalent to the same phase-change process with a temperature boundary condition on the fixed face $x=0$ studied in [27] and therefore an inequality 
for the coefficient which characterized one of the two free interfaces is also obtained in Sections 4 and 6. Moreover, in Section 3, we obtain the convergence of the solution of the phase-change process with a convective boundary condition to the solution given in [27] for a temperature boundary condition at the fixed face $x=0$ when the heat transfer coefficient goes to infinity, and we also give the order of the corresponding convergence when the coefficient that characterized the transient heat transfer at $x=0$ goes to infinity.

This paper completes the model given in [27] by considering two new boundary conditions (convective and heat flux) at the fixed face of the PCMs and obtaining explicit solutions for both cases when a restriction on data is satisfied.

\section{Explicit Solution with a Convective Boundary Condition}

The phase-change process consists in finding the free boundaries $x=s(t)$ and $x=r(t)$ and the temperature $T=T(x, t)$ such that the following conditions must be verified (problem $\left.\left(\mathrm{P}_{1}\right)\right)$ :

$$
\begin{aligned}
& T_{t}-\alpha T_{x x}=0, \\
& \quad 0<x<s(t), t>0\left(\alpha=\frac{k}{\rho c}\right), \\
& T(s(t), t)=0, \quad t>0, \\
& k T_{x}(s(t), t)=\rho \ell[\varepsilon \dot{s}(t)+(1-\varepsilon) \dot{r}(t)], \\
& T_{x}(s(t), t)(r(t)-s(t))=\gamma>0, \quad t>0(\text { with } \gamma>0), \\
& s(0)=r(0)=0, \\
& k T_{x}(0, t)=\frac{h_{0}}{\sqrt{t}}\left(T(0, t)+D_{\infty}\right), \\
& \quad t>0\left(h_{0}>0, D_{\infty}>0\right) .
\end{aligned}
$$

Condition (6) represents a convective boundary condition (Robin condition) at the fixed face $x=0$ [31-33] with a heat transfer coefficient which is inversely proportional to the square root of the time $[29,30,34,35]$. Now, we will obtain the solution of problem (1)-(6) when data satisfy the restriction (7).

Theorem 1. If the coefficient $h_{0}$ satisfies the inequality

$$
h_{0}>\frac{1}{D_{\infty}} \sqrt{\frac{\gamma(1-\varepsilon) \rho \ell k}{2}}=h_{0}^{*},
$$

then the solution of problem (1)-(6) is given by

$$
\begin{array}{r}
T(x, t) \\
=-\frac{\left(h_{0} D_{\infty} \sqrt{\pi \alpha} / k\right) \operatorname{erf}(\xi)}{1+\left(h_{0} \sqrt{\pi \alpha} / k\right) \operatorname{erf}(\xi)}\left[\begin{array}{r}
\operatorname{erf}(x / 2 \sqrt{\alpha t}) \\
\operatorname{erf}(\xi)
\end{array}\right], \\
0<x<s(t), t>0,
\end{array}
$$

$$
\begin{aligned}
& s(t)=2 \xi \sqrt{\alpha t}, \quad t>0, \\
& r(t)=2 \mu \sqrt{\alpha t}, \quad t>0,
\end{aligned}
$$

with

$$
\mu=\xi+\frac{\gamma k}{2 D_{\infty} h_{0} \sqrt{\alpha}} e^{\xi^{2}}\left[1+\frac{h_{0} \sqrt{\pi \alpha}}{k} \operatorname{erf}(\xi)\right]
$$

and the coefficient $\xi$ is given as the unique solution of the equation

$$
\frac{D_{\infty} c}{\ell \sqrt{\pi}} F(x)=G(x), \quad x>0
$$

where the real functions $G$ and $F$ are defined by

$$
\begin{aligned}
& F(x)=\frac{e^{-x^{2}}}{k / h_{0} \sqrt{\pi \alpha}+\operatorname{erf}(x)}, \\
& G(x)=x+\frac{\gamma(1-\varepsilon) \sqrt{\pi}}{2 D_{\infty}} \frac{1}{F(x)},
\end{aligned}
$$

Proof. Taking into account that $\operatorname{erf}(x / 2 \sqrt{\alpha t})$ is a solution of the heat equation (3) [3], we propose as a solution of problem (1)-(6) the following expression:

$$
\begin{aligned}
T(x, t)=C_{1}+C_{2} \operatorname{erf}\left(\frac{x}{2 \sqrt{\alpha t}}\right), & \\
& 0<x<s(t), t>0,
\end{aligned}
$$

where the two coefficients $C_{1}$ and $C_{2}$ must be determined.

From condition (4), we deduce the expression (9) for the free boundary $s(t)$, where the coefficient $\xi$ must be determined. From conditions (6) and (2), we deduce the system of equations

$$
\begin{aligned}
C_{2} & =\frac{h_{0} \sqrt{\pi \alpha}}{k}\left(C_{1}+D_{\infty}\right), \\
C_{1}+C_{2} \operatorname{erf}(\xi) & =0
\end{aligned}
$$

whose solution is given by

$$
\begin{aligned}
& C_{1}=-\frac{\left(h_{0} \sqrt{\pi \alpha} / k\right) D_{\infty} \operatorname{erf}(\xi)}{1+\left(h_{0} \sqrt{\pi \alpha} / k\right) \operatorname{erf}(\xi)}, \\
& C_{2}=\frac{h_{0} D_{\infty} \sqrt{\pi \alpha}}{k} \frac{1}{1+\left(h_{0} \sqrt{\pi \alpha} / k\right) \operatorname{erf}(\xi)},
\end{aligned}
$$

and then we get expression (8) for the temperature.

From condition (4), we deduce expression (10) for the interface $r(t)$ and expression (11) for $\mu$. From condition (3), 
we deduce (12) for the coefficient $\xi$. Functions $F_{3}$ and $G$ have the following properties:

$$
\begin{aligned}
F\left(0^{+}\right) & =\frac{h_{0} \sqrt{\pi \alpha}}{k}>0, \\
F(+\infty) & =0^{+}, \\
F^{\prime}(x) & <0, \quad \forall x>0, \\
G\left(0^{+}\right) & =\frac{(1-\varepsilon) \gamma k}{2 D_{\infty} h_{0} \sqrt{\alpha}}>0, \\
G(+\infty) & =+\infty, \\
G^{\prime}(x) & >0, \quad \forall x>0 .
\end{aligned}
$$

Therefore, we deduce that (12) has a unique solution when the coefficient $h_{0}$ satisfies the inequality

$$
\begin{aligned}
\frac{D_{\infty} c}{\ell \sqrt{\pi}} F\left(0^{+}\right) & >G\left(0^{+}\right) \Longleftrightarrow \\
h_{0}^{2} & >\frac{\gamma(1-\varepsilon) \rho \ell k}{2 D_{\infty}^{2}} ;
\end{aligned}
$$

that is, inequality (7) holds.

Corollary 2. If the coefficient $h_{0}$ satisfies inequality (7), then the temperature, defined by (8), verifies the following inequalities:

$$
-D_{\infty}<T(0, t) \leq T(x, t)<0, \quad 0<x<s(t), t>0 .
$$

Proof. From (8), we obtain

$$
\begin{aligned}
T(0, t) & =-\frac{\left(h_{0} D_{\infty} \sqrt{\pi \alpha} / k\right) \operatorname{erf}(\xi)}{1+\left(h_{0} \sqrt{\pi \alpha} / k\right) \operatorname{erf}(\xi)} \\
& =-\frac{D_{\infty}}{1+k / h_{0} \sqrt{\pi \alpha} \operatorname{erf}(\xi)}>-D_{\infty}, \quad \forall t>0 .
\end{aligned}
$$

Moreover, from (8) and (20), we also get

$$
\begin{aligned}
& T(x, t)+D_{\infty} \\
& =\frac{D_{\infty}}{1+\left(h_{0} \sqrt{\pi \alpha} / k\right) \operatorname{erf}(\xi)}\left[1+\frac{h_{0} \sqrt{\pi \alpha}}{k} \operatorname{erf}\left(\frac{x}{2 \sqrt{\alpha t}}\right)\right] \\
& \geq \frac{D_{\infty}}{1+\left(h_{0} \sqrt{\pi \alpha} / k\right) \operatorname{erf}(\xi)}=T(0, t)+D_{\infty}>0, \\
& \quad 0<x<s(t), t>0 ;
\end{aligned}
$$

that is, (19) holds.

\section{Asymptotic Behavior When the Coefficient $h_{0} \rightarrow+\infty$}

Now, we will obtain the asymptotic behaviour of solution (8)(12) of problem (1)-(6) when the heat transfer coefficient is large, that is, when $h_{0} \rightarrow+\infty$. From a physical point of view, it must be convergent to the solution of the same problem with a temperature boundary condition at the fixed face $x=0$ given by (23).

For any coefficient $h_{0}$ satisfying inequality (7), we will denote the temperature $T$ and the two free boundaries $s$ and $r$ (defined in (8), (9), and (10), resp.) by $T=T\left(x, t, h_{0}\right)$, $x=s\left(t, h_{0}\right)$, and $x=r\left(t, h_{0}\right)$, respectively, with coefficients $\xi=\xi\left(h_{0}\right)$ and $\mu=\mu\left(h_{0}\right)$. We will also denote by $F\left(x, h_{0}\right)$ and $G\left(x, h_{0}\right)$ the functions defined in (13). We have the following result.

Theorem 3. One obtains the following limits:

$$
\begin{aligned}
\lim _{h_{0} \rightarrow \infty} T\left(x, t, h_{0}\right) & =T_{\infty}(x, t), \\
\lim _{h_{0} \rightarrow \infty} s\left(t, h_{0}\right) & =s_{\infty}(t), \\
\lim _{h_{0} \rightarrow \infty} r\left(t, h_{0}\right) & =r_{\infty}(t),
\end{aligned}
$$

where $T_{\infty}(x, t), s_{\infty}(t)$, and $r_{\infty}(t)$ are the solutions of the following phase-change process with mushy region: (1)-(5) and

$$
T(0, t)=-D_{\infty}, \quad t>0,
$$

instead of the boundary condition (6).

Proof. The solution of problem (1)-(5) and (23) is given by [27]

$$
\begin{gathered}
T_{\infty}(x, t)=-D_{\infty}\left[1-\frac{\operatorname{erf}(x / 2}{\operatorname{erf}(\xi}\right. \\
s_{\infty}(t)=2 \xi_{\infty} \sqrt{\alpha t}, \quad t>0, \\
r_{\infty}(t)=2 \mu_{\infty} \sqrt{\alpha t}, \quad t>0,
\end{gathered}
$$

with

$$
\mu_{\infty}=\xi_{\infty}+\frac{\gamma \sqrt{\pi}}{2 D_{\infty}} e^{\xi_{\infty}^{2}} \operatorname{erf}\left(\xi_{\infty}\right)
$$

and the coefficient $\xi_{\infty}$ given as the unique solution of the equation

$$
G_{1}(x)=\frac{D_{\infty} c}{\ell \sqrt{\pi}}, \quad x>0,
$$

where the real function $G_{1}$ is defined by

$$
G_{1}(x)=\frac{G_{\infty}(x)}{F_{\infty}(x)}, \quad x>0,
$$

with

$$
G_{\infty}(x)=x+\frac{\gamma(1-\varepsilon) \sqrt{\pi}}{2 D_{\infty}} \frac{1}{F_{\infty}(x)}=\lim _{h_{0} \rightarrow \infty} G\left(x, h_{0}\right),
$$

$$
x>0 \text {, }
$$

$$
F_{\infty}(x)=\frac{e^{-x^{2}}}{\operatorname{erf}(x)}=\lim _{h_{0} \rightarrow \infty} F\left(x, h_{0}\right), \quad x>0 .
$$


Then,

$$
\begin{aligned}
& \lim _{h_{0} \rightarrow \infty} \xi\left(h_{0}\right)=\xi_{\infty}, \\
& \lim _{h_{0} \rightarrow \infty} \mu\left(h_{0}\right)=\mu_{\infty} .
\end{aligned}
$$

And, therefore, the limits (22) hold.

Now, by studying the real functions $F\left(x, h_{0}\right)$ and $G\left(x, h_{0}\right)$ as functions of the variable $h_{0}$, we can obtain the order of the convergence of solution (8)-(12) of problem (1)-(6) to solution (24)-(28) of problem (1)-(5) and (23) when $h_{0} \rightarrow$ $\infty$.

Theorem 4. When the variable $h_{0} \rightarrow \infty$, one obtains the following estimations:

$$
\begin{aligned}
& 0<\xi_{\infty}-\xi\left(h_{0}\right)=O\left(\frac{1}{h_{0}}\right) \text { when } h_{0} \longrightarrow \infty \\
& 0<s_{\infty}(t)-s\left(t, h_{0}\right)=O\left(\frac{1}{h_{0}}\right),
\end{aligned}
$$

$$
\forall t \geq 0 \text { when } h_{0} \longrightarrow \infty
$$

$$
\begin{aligned}
& \left|\mu\left(h_{0}\right)-\mu_{\infty}\right|=O\left(\frac{1}{h_{0}}\right) \text { when } h_{0} \rightarrow \infty, \\
& \left|r\left(t, h_{0}\right)-r_{\infty}(t)\right|=O\left(\frac{1}{h_{0}}\right),
\end{aligned}
$$

$$
\forall t \geq 0 \text { when } h_{0} \longrightarrow \infty
$$

$$
\left|T\left(x, t, h_{0}\right)-T_{\infty}(x, t)\right|=O\left(\frac{1}{h_{0}}\right)
$$

$$
\forall x \geq 0, \forall t>0 \text { when } h_{0} \longrightarrow \infty \text {. }
$$

Proof. As the variable $h_{0} \rightarrow \infty$, we can consider that $h_{0}>h_{0}^{*}$ and then solution (8)-(12) of problem (1)-(6) is well defined.

Function $F\left(x, h_{0}\right)$ is an increasing function in variable $h_{0}$; therefore, function $G\left(x, h_{0}\right)$ is a decreasing function in variable $h_{0}$. Then, function $G\left(x, h_{0}\right) / F\left(x, h_{0}\right)$ is a decreasing function in variable $h_{0}, \forall x>0$, which is convergent to $G_{\infty}(x) / F_{\infty}(x)$ as $h_{0} \rightarrow \infty$ because (30) and (31) hold.

By using (13) and (31), we have

$$
\begin{aligned}
0 & <F_{\infty}(x)-F\left(x, h_{0}\right)=F_{\infty}(x) \frac{k / h_{0} \sqrt{\pi \alpha}}{\operatorname{erf}(x)+k / h_{0} \sqrt{\pi \alpha}} \\
& <\frac{e^{-x^{2}}}{\operatorname{erf}^{2}(x)} \frac{k}{h_{0} \sqrt{\pi \alpha}}=O\left(\frac{1}{h_{0}}\right),
\end{aligned}
$$

By using (13) and (30), we have

$$
\begin{aligned}
& 0<G\left(x, h_{0}\right)-G_{\infty}(x) \\
&=\frac{\gamma(1-\varepsilon) \sqrt{\pi}}{2 D_{\infty}} \frac{F_{\infty}(x)-F\left(x, h_{0}\right)}{F_{\infty}(x) F\left(x, h_{0}\right)} \\
&<\frac{\gamma(1-\varepsilon) \sqrt{\pi}}{2 D_{\infty}} \frac{F_{\infty}(x)-F\left(x, h_{0}\right)}{F_{\infty}(x) F\left(x, h_{0}^{*}\right)}=O\left(\frac{1}{h_{0}}\right), \\
& \forall x>0 \text { when } h_{0} \rightarrow \infty .
\end{aligned}
$$

Therefore, we have

$$
\begin{aligned}
0< & \frac{G\left(x, h_{0}\right)}{F\left(x, h_{0}\right)}-\frac{G_{\infty}(x)}{F_{\infty}(x)}<\left[F_{\infty}(x)-F\left(x, h_{0}\right)\right] \\
& \frac{x+\left(\gamma(1-\varepsilon) \sqrt{\pi} / D_{\infty}\right)\left(1 / F\left(x, h_{0}^{*}\right)\right)}{F_{\infty}(x) F\left(x, h_{0}^{*}\right)} \\
& =O\left(\frac{1}{h_{0}}\right), \quad \forall x>0 \text { when } h_{0} \longrightarrow \infty .
\end{aligned}
$$

Then, the estimation (33) holds and

$$
\begin{aligned}
0 & <s_{\infty}(t)-s\left(t, h_{0}\right)=2 \sqrt{\alpha t}\left(\xi_{\infty}-\xi\left(h_{0}\right)\right) \\
& =O\left(\frac{1}{h_{0}}\right), \quad \forall t \geq 0 \text { when } h_{0} \longrightarrow \infty .
\end{aligned}
$$

By using (11) and (27), we get

$$
\begin{aligned}
& \mu_{\infty}-\mu\left(h_{0}\right) \\
& =\left(\xi_{\infty}-\xi\left(h_{0}\right)\right)-\frac{\gamma \sqrt{\pi}}{2 D_{\infty} \sqrt{\alpha}} \frac{e^{\xi^{2}\left(h_{0}\right)}}{h_{0}} \\
& \quad+\frac{\gamma \sqrt{\pi}}{2 D_{\infty}}\left[e^{\xi_{\infty}^{2}} \operatorname{erf}\left(\xi_{\infty}\right)-e^{\xi^{2}\left(h_{0}\right)} \operatorname{erf}\left(\xi\left(h_{0}\right)\right)\right] \\
& =O\left(\frac{1}{h_{0}}\right) \quad \text { when } h_{0} \longrightarrow \infty, \\
& \left|r\left(t, h_{0}\right)-r_{\infty}(t)\right|=2 \sqrt{\alpha t}\left|\mu\left(h_{0}\right)-\mu_{\infty}\right|=O\left(\frac{1}{h_{0}}\right), \\
& \forall t \geq 0 \text { when } h_{0} \longrightarrow \infty .
\end{aligned}
$$

Finally, by using (8) and (24), we get

$$
\begin{aligned}
& T\left(x, t, h_{0}\right)-T_{\infty}(x, t) \\
& \quad=\frac{D_{\infty}}{1+\left(h_{0} \sqrt{\pi \alpha} / k\right) \operatorname{erf}\left(\xi\left(h_{0}\right)\right)}\{1 \\
& \quad+\frac{\operatorname{erf}(x / 2 \sqrt{\alpha t})}{\operatorname{erf}\left(\xi_{0}\right)}\left[\frac { h _ { 0 } \sqrt { \pi \alpha } } { k } \left(\operatorname{erf}\left(\xi_{\infty}\right)\right.\right. \\
& \left.\left.\left.-\operatorname{erf}\left(\xi\left(h_{0}\right)\right)\right)\right]\right\}=O\left(\frac{1}{h_{0}}\right),
\end{aligned}
$$


and the thesis holds. In the particular case, when $x=0$, we have

$$
\begin{aligned}
0 & <T\left(0, t, h_{0}\right)-T_{\infty}(0, t) \\
& =\frac{D_{\infty}}{1+\left(h_{0} \sqrt{\pi \alpha} / k\right) \operatorname{erf}\left(\xi\left(h_{0}\right)\right)} \\
& <\frac{k D_{\infty}}{h_{0} \sqrt{\pi \alpha} \operatorname{erf}\left(\xi\left(h_{0}^{*}\right)\right)}=O\left(\frac{1}{h_{0}}\right),
\end{aligned}
$$

$$
\forall t>0 \text { when } h_{0} \longrightarrow \infty \text {. }
$$

\section{Equivalence between the Mushy Zone Models with Convective and Temperature Boundary Conditions}

We consider problem $\left(\mathrm{P}_{2}\right)$ defined by conditions (1)-(5) and temperature boundary condition

$$
T(0, t)=-D_{0}<0, \quad t>0,
$$

at the fixed face $x=0$, whose solution was given in [27]. We have the following property.

Theorem 5. If the coefficient $h_{0}$ satisfies inequality (7), then problem $\left(P_{1}\right)$, defined by conditions (1)-(6), is equivalent to problem $\left(P_{2}\right)$, defined by conditions (1)-(5) and (45), when the parameter $D_{0}$ in problem $\left(P_{2}\right)$ is related to parameters $h_{0}$ and $D_{0}$ in problem $\left(P_{1}\right)$ by the following expression:

$$
D_{0}=\frac{D_{\infty} \operatorname{erf}(\xi)}{k / h_{0} \sqrt{\pi \alpha}+\operatorname{erf}(\xi)}>0,
$$

where the coefficient $\xi$ is given as the unique solution of (12) for problem $\left(P_{1}\right)$ or as the unique solution of equation

$$
G_{2}(x)=\frac{D_{0} c}{\ell \sqrt{\pi}}, \quad x>0,
$$

for problem $\left(P_{2}\right)$, where the real function $G_{2}$ is defined by

$$
\begin{aligned}
G_{2}(x) & =\frac{G_{0}(x)}{F_{\infty}(x)}, \\
G_{0}(x) & =\left[x+\frac{\gamma(1-\varepsilon) \sqrt{\pi}}{2 D_{0}} \frac{1}{F_{\infty}(x)}\right],
\end{aligned}
$$

$$
x>0 .
$$

Proof. If the coefficient $h_{0}$ satisfies inequality (7), then the solution of problem $\left(\mathrm{P}_{1}\right)$ is given by $(8)-(12)$. Taking into account that

$$
\begin{aligned}
T(0, t) & =-\frac{\left(h_{0} \sqrt{\pi} \alpha / k\right) D_{\infty} \operatorname{erf}(\xi)}{1+\left(h_{0} \sqrt{\pi} \alpha / k\right) \operatorname{erf}(\xi)} \\
& =-\frac{D_{\infty} \operatorname{erf}(\xi)}{k / h_{0} \sqrt{\pi} \alpha+\operatorname{erf}(\xi)}<0, \quad t>0,
\end{aligned}
$$

we can define problem $\left(\mathrm{P}_{2}\right)$ by imposing the temperature boundary condition (45) with data $D_{0}$ given in (46). By using this data $D_{0}$ in problem $\left(\mathrm{P}_{2}\right)$ and the method developed in [30], we can prove that the solutions of both problems $\left(P_{1}\right)$ and $\left(P_{2}\right)$ are the same and then the two problems are equivalent.

Corollary 6. If the coefficient $h_{0}$ satisfies inequality (7), then the coefficient $\xi$ of the solid-mushy zone interface of problem $\left(P_{2}\right)$ verifies the following inequality:

$$
\operatorname{erf}(\xi)<\frac{D_{\infty} D_{0}}{D_{\infty}-D_{0}} \sqrt{\frac{2 c}{\pi \gamma(1-\varepsilon) \ell}}, \quad \forall D_{\infty}>D_{0}
$$

Then,

$$
\operatorname{erf}(\xi)<D_{0} \sqrt{\frac{2 c}{\pi \gamma(1-\varepsilon) \ell}}
$$

Remark 7. The real functions $G_{\infty}$, defined in (30), and $G_{0}$, defined in (48), are similar; the difference between them is the parameters $D_{\infty}$ or $D_{0}$ used in each definition.

\section{Explicit Solution with a Heat Flux Boundary Condition}

Now, we will consider a phase-change process which consists in finding the free boundaries $x=s(t)$ and $x=r(t)$ and the temperature $T=T(x, t)$ such that the following conditions must be verified (problem $\left(\mathrm{P}_{3}\right)$ ): conditions (1)-(5) and

$$
k T_{x}(0, t)=\frac{q_{0}}{\sqrt{t}}, \quad t>0\left(q_{0}>0\right) .
$$

Condition (52) represents the heat flux at the fixed face $x=0$ characterized by a coefficient which is inversely proportional to the square root of the time [34].

Theorem 8. If the coefficient $q_{0}$ satisfies the inequality

$$
q_{0}>\sqrt{\frac{\gamma(1-\varepsilon) \rho \ell k}{2}}=q_{0}^{*}
$$

then the solution of problem (1)-(5) and (52) is given by

$$
\begin{aligned}
& T(x, t)=-\frac{q_{0} \sqrt{\pi \alpha} \operatorname{erf}(\omega)}{k}\left[1-\frac{\operatorname{erf}(x / 2 \sqrt{\alpha t})}{\operatorname{erf}(\omega)}\right]<0, \\
& 0<x<s(t), t>0, \\
& s(t)=2 \omega \sqrt{\alpha t}, \quad t>0, \\
& r(t)=2 v \sqrt{\alpha t}, \quad t>0,
\end{aligned}
$$

with

$$
v=\omega+\frac{\gamma k}{2 q_{0} \sqrt{\alpha}} e^{\omega^{2}}
$$


and the coefficient $\omega>0$ given as the unique solution of the equation

$$
G_{3}(x)=\frac{q_{0}}{\rho \ell \sqrt{\alpha}}, \quad x>0,
$$

where the real function $G_{3}$ is defined by

$$
G_{3}(x)=\left[x+\frac{\gamma(1-\varepsilon) k}{2 q_{0} \sqrt{\alpha}} e^{x^{2}}\right] e^{x^{2}}, \quad x>0 .
$$

Proof. Following the proof of Theorem 1, we propose as a solution of problem (1)-(5) and (52) the following expression:

$$
\begin{aligned}
T(x, t)=A_{1}+A_{2} \operatorname{erf}\left(\frac{x}{2 \sqrt{\alpha t}}\right) & \\
& 0<x<s(t), t>0,
\end{aligned}
$$

where the two coefficients $A_{1}$ and $A_{2}$ must be determined.

From condition (2), we deduce expression (55) for the free boundary $s(t)$, with the coefficient $\omega$ to be determined. From conditions (2) and (52), we deduce

$$
\begin{aligned}
& A_{1}=-\frac{q_{0} \sqrt{\pi \alpha}}{k} \operatorname{erf}(\omega), \\
& A_{2}=\frac{q_{0} \sqrt{\pi \alpha}}{k},
\end{aligned}
$$

and then we get expression (54) for the temperature.

From condition (4), we deduce expression (56) for the interface $r(t)$ and expression (57) for $\nu$. From condition (3), we deduce (58) for the coefficient $\omega$. Since function $G_{3}$ has the following properties:

$$
\begin{aligned}
G_{3}\left(0^{+}\right) & =\frac{\gamma(1-\varepsilon) k}{2 q_{0} \sqrt{\alpha}}>0, \\
G_{3}(+\infty) & =+\infty, \\
G_{3}^{\prime}(x) & >0, \quad \forall x>0,
\end{aligned}
$$

we can deduce that (58) has a unique solution when the coefficient $q_{0}$ satisfies the inequality

$$
\begin{aligned}
\frac{q_{0}}{\rho \ell \sqrt{\alpha}}>G_{3}\left(0^{+}\right) \Longleftrightarrow \\
q_{0}^{2}>\frac{\gamma(1-\varepsilon) \rho \ell k}{2},
\end{aligned}
$$

which is inequality (53).

Remark 9. We have a relationship between $q_{0}^{*}$ (the lower limit for coefficient $q_{0}$ in order to have a phase-change process with a mushy region with a heat flux boundary condition at $x=0$ ) and $h_{0}^{*}$ (the lower limit for the coefficient $h_{0}$ in order to have a phase-change process with a mushy region with a convective boundary condition at $x=0$ ) given by

$$
q_{0}^{*}=D_{\infty} h_{0}^{*} .
$$

\section{Equivalence between the Mushy Zone Models with Heat Flux and Temperature Boundary Conditions}

Following Section 4, we will now study the relationship between problems $\left(\mathrm{P}_{3}\right)$ and $\left(\mathrm{P}_{2}\right)$. We have the following property.

Theorem 10. If the coefficient $q_{0}$ satisfies inequality (53), then problem $\left(P_{3}\right)$, defined by conditions (1)-(5) and (52), is equivalent to problem $\left(P_{2}\right)$, defined by conditions (1)-(5) and (45), when the parameter $D_{0}$ in problem $\left(P_{2}\right)$ is related to the parameter $q_{0}$ in problem $\left(P_{3}\right)$ by the following expression:

$$
D_{0}=\frac{q_{0} \sqrt{\pi \alpha}}{k} \operatorname{erf}(\omega)>0
$$

where the coefficient $\omega$ is given as the unique solution of (58) for problem $\left(P_{3}\right)$ or as the unique solution of (47) for problem $\left(P_{2}\right)$.

Proof. If the coefficient $q_{0}$ satisfies inequality (53), then the solution of problem $\left(\mathrm{P}_{3}\right)$ is given by $(54)-(58)$. Taking into account that

$$
T(0, t)=-\frac{q_{0} \sqrt{\pi \alpha}}{k} \operatorname{erf}(\omega)<0, \quad t>0,
$$

we can define problem $\left(\mathrm{P}_{2}\right)$ by imposing the temperature boundary condition (45) with the data $D_{0}$ given in (65). By using this data $D_{0}$ in problem $\left(\mathrm{P}_{2}\right)$ and the method developed in [30], we can prove that the solutions of both problems $\left(\mathrm{P}_{3}\right)$ and $\left(\mathrm{P}_{2}\right)$ are the same and then the two problems are equivalent.

Corollary 11. If the coefficient $q_{0}$ satisfies inequality (53), then the coefficient $\xi$ of the solid-mushy zone interface of problem $\left(P_{2}\right)$ verifies inequality $(51)$ which is the same as that we have obtained through the equivalence between problems $\left(P_{1}\right)$ and $\left(P_{2}\right)$.

Remark 12. At last, for a suggestion of an anonymous referee, we will transform problem $\left(\mathrm{P}_{1}\right)$, given by the equations and conditions (1)-(6), and inequality (7) in a dimensionless form. We define the following dimensionless change of variables:

$$
\begin{aligned}
\eta & =\frac{x}{L}, \\
\tau & =\frac{\alpha t}{L^{2}}, \\
S(\tau) & =\frac{s(t)}{L}, \\
R(\tau) & =\frac{r(t)}{L}, \\
\theta(\eta, \tau) & =\frac{T(x, t)}{D_{\infty}},
\end{aligned}
$$


where $L$ is a characteristic length. Therefore, the equations and conditions (1)-(6) are transformed as

$$
\begin{aligned}
& \theta_{\tau}-\theta_{\eta \eta}=0, \quad 0<\eta<S(\tau), \tau>0, \\
& \theta(S(\tau), \tau)=0, \quad \tau>0, \\
& \theta_{\eta}(r(\tau), \tau)=\frac{1}{\operatorname{Ste}}\left[\varepsilon S^{\prime}(\tau)+(1-\varepsilon) R^{\prime}(\tau)\right], \quad \tau>0, \\
& \theta_{\eta}(r(\tau), \tau)(R(\tau)-S(\tau))=\frac{\gamma}{D_{\infty}}, \quad \tau>0, \\
& S(0)=R(0)=0, \\
& \theta_{\eta}(0, \tau)=\frac{B}{\sqrt{\tau}}(\theta(0, \tau)+1), \quad \tau>0,
\end{aligned}
$$

where Ste is the Stefan number and $B / \sqrt{\tau}$ is the Biot number defined by the following expressions:

$$
\begin{aligned}
\text { Ste } & =\frac{c D_{\infty}}{\ell}>0, \\
B & =\frac{h_{0} \sqrt{\alpha}}{k}=\frac{h_{0}}{\sqrt{\rho k c}}>0 .
\end{aligned}
$$

Moreover, inequality (7) for the physical coefficient $h_{0}$, which characterized the heat transfer coefficient in the boundary condition (6), is transformed in the following way:

$$
B>\frac{1}{D_{\infty}} \sqrt{\frac{\gamma(1-\varepsilon) \ell}{2 c}}=B^{*} .
$$

Therefore, limit $h_{0} \rightarrow \infty$ in problem (1)-(6) in physical variables is equivalent to limit $B \rightarrow \infty$ in problem (68) in dimensionless variables.

By using the results of this work, we can now obtain new explicit expression for the determination of one or two unknown thermal coefficients through a phase-change process with a mushy zone by imposing an overspecified convective boundary condition at the fixed face $x=0$. This will complete and improve the results obtained previously in [28].

\section{Conclusions}

The goal of this paper is to complete the solution of SolomonWilson-Alexiades's model for a mushy zone model for phasechange materials when a convective or a heat flux boundary condition at the fixed face $x=0$ is imposed. In both cases, explicit solutions for the temperature and the two free boundaries which define the mushy region were obtained and, for both cases, the necessary and sufficient conditions on data in order to get these explicit solutions are also obtained which is new with respect to the original model when a temperature boundary condition at the face $x=0$ was imposed. Moreover, the equivalence of this two phase-change

\begin{tabular}{|c|c|}
\hline$B\left(B^{*}\right):$ & $\begin{array}{l}\text { Dimensionless coefficient that } \\
\text { characterizes the transient heat } \\
\text { transfer at } x=0 \text { (Biot number), } \\
\text { defined in }(70)\end{array}$ \\
\hline$c:$ & Specific heat, $J /\left(\mathrm{kg}^{\circ} \mathrm{C}\right)$ \\
\hline$-D_{0}(<0):$ & $\begin{array}{l}\text { Temperature at the fixed face } x=0 \text {, } \\
{ }^{\circ} \mathrm{C}\end{array}$ \\
\hline$-D_{\infty}(<0):$ & $\begin{array}{l}\text { Bulk temperature at the fixed face } \\
x=0,{ }^{\circ} \mathrm{C}\end{array}$ \\
\hline$h_{0}\left(h_{0}^{*}\right):$ & $\begin{array}{l}\text { Coefficient that characterizes the } \\
\text { transient heat transfer at } x=0 \text {, } \\
\mathrm{kg} /\left(\mathrm{C}^{\circ} \mathrm{s}^{5 / 2}\right)\end{array}$ \\
\hline$k:$ & Thermal conductivity, $\mathrm{W} /\left(\mathrm{m}^{\circ} \mathrm{C}\right)$ \\
\hline$\ell:$ & $\begin{array}{l}\text { Latent heat of fusion by unit of mass, } \\
\mathrm{J} / \mathrm{kg}\end{array}$ \\
\hline$L:$ & Characteristic length, $\mathrm{m}$ \\
\hline $\mathrm{P}_{1}:$ & $\begin{array}{l}\text { Phase-change process defined by } \\
\text { conditions }(1)-(6)\end{array}$ \\
\hline $\mathrm{P}_{2}$ : & $\begin{array}{l}\text { Phase-change process defined by } \\
\text { conditions (1)-(5) and (45) }\end{array}$ \\
\hline $\mathrm{P}_{3}$ : & $\begin{array}{l}\text { Phase-change process defined by } \\
\text { conditions (1)-(5) and (52) }\end{array}$ \\
\hline$q_{0}\left(q_{0}^{*}\right):$ & $\begin{array}{l}\text { Coefficient that characterizes the } \\
\text { transient heat flux at } x=0, \mathrm{~kg} / \mathrm{s}^{5 / 2}\end{array}$ \\
\hline$r=r(t)(>s(t)):$ & $\begin{array}{l}\text { Position of the liquid-mushy zone } \\
\text { interface at time } t, \mathrm{~m}\end{array}$ \\
\hline$R=R(\tau)(>S(\tau))$ & $\begin{array}{l}\text { Dimensionless position of the } \\
\text { liquid-mushy zone interface at time } \tau\end{array}$ \\
\hline$s=s(t)$ & $\begin{array}{l}\text { Position of the solid-mushy zone } \\
\text { interface at time } t, \mathrm{~m}\end{array}$ \\
\hline$S=S(\tau)$ & $\begin{array}{l}\text { Dimensionless position of the } \\
\text { solid-mushy zone interface at time } \tau\end{array}$ \\
\hline Ste: & Stefan number, defined in (69) \\
\hline$t:$ & Time, s \\
\hline$T:$ & Temperature of the solid phase, ${ }^{\circ} \mathrm{C}$ \\
\hline$x:$ & Spatial coordinate, $\mathrm{m}$. \\
\hline
\end{tabular}
process with the one with a temperature boundary condition on the fixed face $x=0$ was obtained and an inequality for the dimensionless coefficient that characterizes the first free boundary is also given. On the other hand, the convergence of the phase-change process with mushy zone when the heat transfer coefficient goes to infinity was also obtained and the order of the convergence is also shown.

\section{Nomenclature}

Greek Symbols

$\alpha=k / \rho c:$ Diffusivity coefficient, $\mathrm{m}^{2} / \mathrm{s}$

$\gamma>0: \quad$ One of the two coefficients that characterizes the mushy zone, ${ }^{\circ} \mathrm{C}$

$\varepsilon \in(0,1)$ : One of the two coefficients that characterizes the mushy zone, being dimensionless

$\nu(>\omega): \quad$ Coefficient that characterizes the free boundary $r(t)$ in (45), being dimensionless

$\rho: \quad$ Density of mass, $\mathrm{kg} / \mathrm{m}^{3}$

$\mu(>\xi): \quad$ Coefficient that characterizes the free boundary $r(t)$ in (10), being dimensionless

$\mu_{\infty}\left(>\xi_{\infty}\right)$ : Coefficient that characterizes the free boundary $r(t)$ in (26), being dimensionless 
$\omega>0$ : Coefficient that characterizes the free boundary $s(t)$ in (43), being dimensionless

$\xi>0$ : Coefficient that characterizes the free boundary $s(t)$ in (9), being dimensionless

$\xi_{\infty}>0$ : Coefficient that characterizes the free boundary $s(t)$ in (25), being dimensionless

$\tau$ : $\quad$ Dimensionless time

$\theta: \quad$ Dimensionless temperature of the solid phase

$\eta: \quad$ Dimensionless spatial coordinate.

\section{Conflict of Interests}

The author declares that there is no conflict of interests regarding the publication of this paper.

\section{Acknowledgments}

The authors would like to thank an anonymous referee for his constructive comments which improves the readability of the paper. The present work has been partially sponsored by the Projects PIP no. 0534 from CONICET, Universidad Austral, Rosario, Argentina and AFOSR-SOARD Grant FA9550-14-10122 .

\section{References}

[1] V. Alexiades and A. D. Solomon, Mathematical Modeling of Melting and Freezing Processes, Hemisphere-Taylor \& Francis, Washington, DC, USA, 1996.

[2] J. R. Cannon, The One-Dimensional Heat Equation, AddisonWesley, Menlo Park, Calif, USA, 1984.

[3] H. S. Carslaw and C. J. Jaeger, Conduction of Heat in Solids, Clarendon Press, Oxford, UK, 1959.

[4] J. Crank, Free and Moving Boundary Problems, Clarendon Press, Oxford, UK, 1984.

[5] A. Fasano, "Mathematical models of some diffusive processes with free boundary," Matematica-Serie A, vol. 11, pp. 1-128, 2005.

[6] S. C. Gupta, The Classical Stefan Problem. Basic Concepts, Modelling and Analysis, Elsevier, Amsterdam, The Netherlands, 2003.

[7] V. J. Lunardini, Heat Transfer with Freezing and Thawing, Elsevier, London, UK, 1991.

[8] L. I. Rubinstein, The Stefan Problem, American Mathematical Society, Providence, RI, USA, 1971.

[9] A. B. Tayler, Mathematical Models in Applied Mechanics, Clarendon Press, Oxford, UK, 1986.

[10] D. A. Tarzia, "A bibliography on moving-free boundary problems for heat diffusion equation. The Stefan problem," MAT Serie A, vol. 2, pp. 1-297, 2000.

[11] N. M. Arifin, R. Nazar, and I. Pop, "Similarity solution of Marangoni convection boundary layer flow over a flat surface in a nanofluid," Journal of Applied Mathematics, vol. 2013, Article ID 634746, 8 pages, 2013.

[12] A. C. Briozzo and D. A. Tarzia, "Explicit solution of a free boundary problem for a nonlinear absorption model of mixed saturated-unsaturated flow," Advances in Water Resources, vol. 21, no. 8, pp. 713-721, 1998.
[13] P. Broadbridge, "Solution of a nonlinear absorption model of mixed saturated-unsaturated flow," Water Resources Research, vol. 26, no. 10, pp. 2435-2443, 1990.

[14] F. Font, S. L. Mitchell, and T. G. Myers, "One-dimensional solidification of supercooled melts," International Journal of Heat and Mass Transfer, vol. 62, no. 1, pp. 411-421, 2013.

[15] Y. Huang and Y. Shang, "The Bäcklund transformations and abundant exact explicit solutions for a general nonintegrable nonlinear convection-diffusion equation," Abstract and Applied Analysis, vol. 2012, Article ID 489043, 11 pages, 2012.

[16] A. Hussanan, I. Khan, and S. Shafie, "An exact analysis of heat and mass transfer past a vertical plate with Newtonian heating," Journal of Applied Mathematics, vol. 2013, Article ID 434571, 9 pages, 2013.

[17] D. Kaya, "Explicit solutions of generalized nonlinear Boussinesq equations," Journal of Applied Mathematics, vol. 1, no. 1, pp. 2937, 2001.

[18] T. G. Myers and F. Font, "On the one-phase reduction of the Stefan problem with a variable phase change temperature," International Communications in Heat and Mass Transfer, vol. 61, pp. 37-41, 2015.

[19] T. G. Myers, S. L. Mitchell, and F. Font, "Energy conservation in the one-phase supercooled Stefan problem," International Communications in Heat and Mass Transfer, vol. 39, no. 10, pp. $1522-1525,2012$.

[20] M. F. Natale and D. A. Tarzia, "Explicit solutions to the twophase Stefan problem for storm-type materials," Journal of Physics A: Mathematical and General, vol. 33, no. 2, pp. 395-404, 2000.

[21] C. Rogers, "On a class of reciprocal Stefan moving boundary problems," Zeitschrift für Angewandte Mathematik und Physik, vol. 66, no. 4, pp. 2069-2079, 2015.

[22] N. N. Salva and D. A. Tarzia, "Explicit solution for a Stefan problem with variable latent heat and constant heat flux boundary conditions," Journal of Mathematical Analysis and Applications, vol. 379, no. 1, pp. 240-244, 2011.

[23] A. Sojoudi, A. Mazloomi, S. C. Saha, and Y. T. Gu, "Similarity solutions for flow and heat transfer of non-Newtonian fluid over a stretching surface," Journal of Applied Mathematics, vol. 2014, Article ID 718319, 8 pages, 2014.

[24] A. D. Solomon, D. G. Wilson, and V. Alexiades, "Explicit solutions to change problems," Quarterly of Applied Mathematics, vol. 41, pp. 237-243, 1983.

[25] V. R. Voller and F. Falcini, "Two exact solutions of a Stefan problem with varying diffusivity," International Journal of Heat and Mass Transfer, vol. 58, no. 1-2, pp. 80-85, 2013.

[26] V. R. Voller, J. B. Swenson, and C. Paola, "An analytical solution for a Stefan problem with variable latent heat," International Journal of Heat and Mass Transfer, vol. 47, no. 24, pp. 5387-5390, 2004.

[27] A. D. Solomon, D. G. Wilson, and V. Alexiades, "A mushy zone model with an exact solution," Letters in Heat and Mass Transfer, vol. 9, no. 4, pp. 319-324, 1982.

[28] D. A. Tarzia, "Determination of unknown thermal coefficients of a semi-infinite material for the one-phase LameClapeyron (Stefan) problem through the Solomon-WilsonAlexiades' mushy zone model," International Communications in Heat and Mass Transfer, vol. 14, no. 2, pp. 219-228, 1987.

[29] D. A. Tarzia, "An explicit solution for a two-phase unidimensional Stefan problem with a convective boundary condition at the fixed face," MAT Serie A, vol. 8, pp. 21-27, 2004. 
[30] D. A. Tarzia, "Relationship between Neumann solutions for two-phase Lamé-Clapeyron-Stefan problems with convective and temperature boundary conditions," Thermal Science, p. 3, 2015.

[31] P. M. Beckett, "A note on surface heat transfer coefficients," International Journal of Heat and Mass Transfer, vol. 34, no. 8, pp. 2165-2166, 1991.

[32] S. D. Foss, "An approximate solution to the moving boundary problem associated with the freezing and melting of lake ice," AIChE Symposium Series, vol. 74, no. 174, pp. 250-255, 1978.

[33] H. Ching-Lun and S. Yen-Ping, "Perturbation solution for planar solidification of a saturated liquid with convection at the wall," International Journal of Heat and Mass Transfer, vol. 18, no. 12, pp. 1481-1483, 1975.

[34] D. A. Tarzia, "An inequality for the coefficient $\sigma$ of the free boundary $s(t)=2 \sigma \sqrt{t}$ of the Neumann solution for the twophase Stefan problem," Quarterly of Applied Mathematics, vol. 39, no. 4, pp. 491-497, 1981.

[35] S. M. Zubair and M. A. Chaudhry, "Exact solutions of solidliquid phase-change heat transfer when subjected to convective boundary conditions," Heat and Mass Transfer, vol. 30, no. 2, pp. 77-81, 1994. 


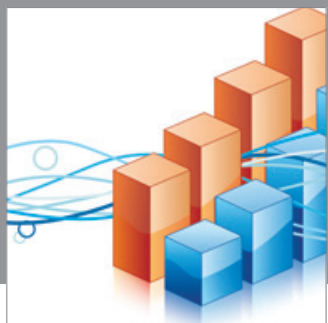

Advances in

Operations Research

mansans

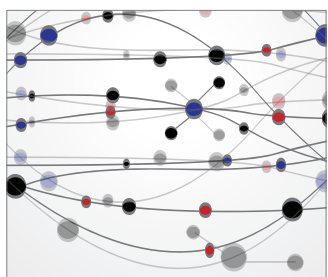

The Scientific World Journal
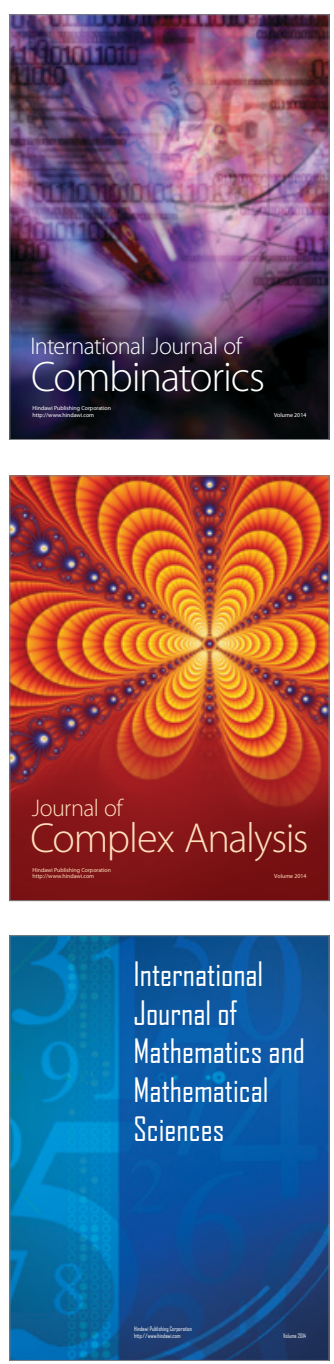
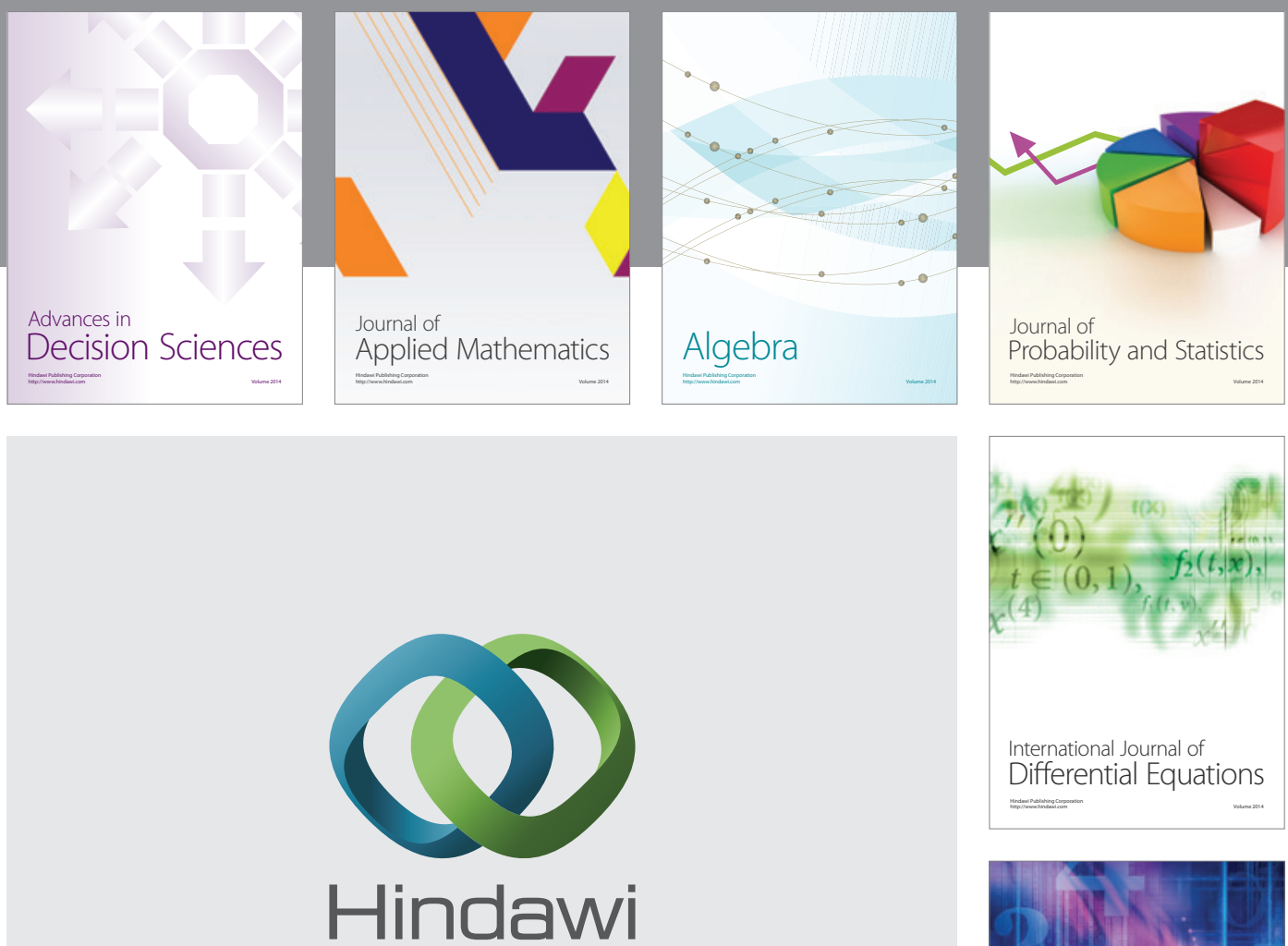

Submit your manuscripts at http://www.hindawi.com
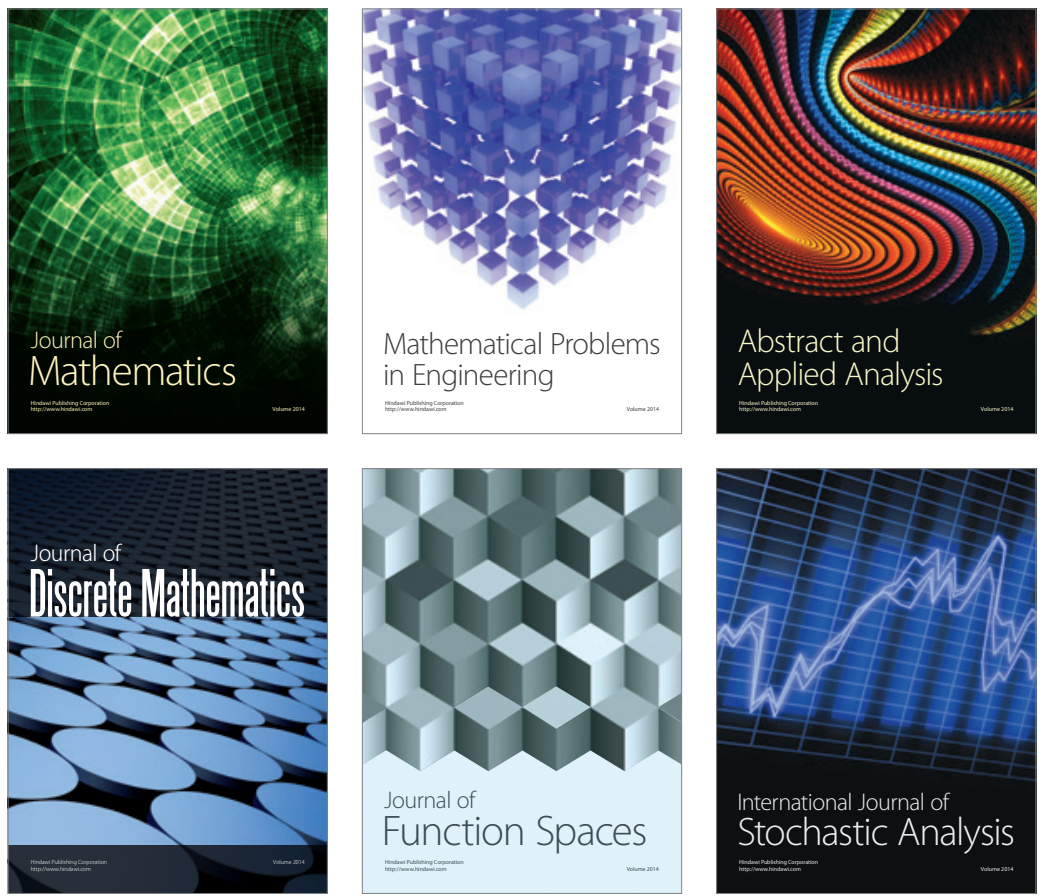

Journal of

Function Spaces

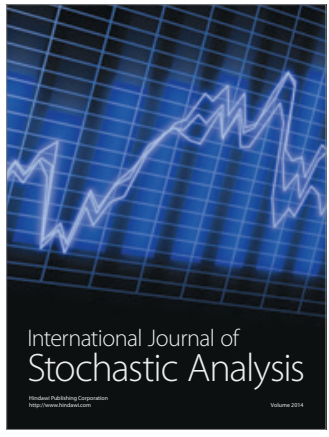

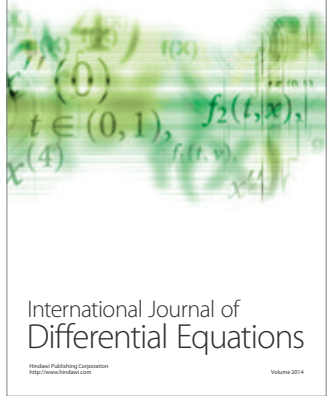
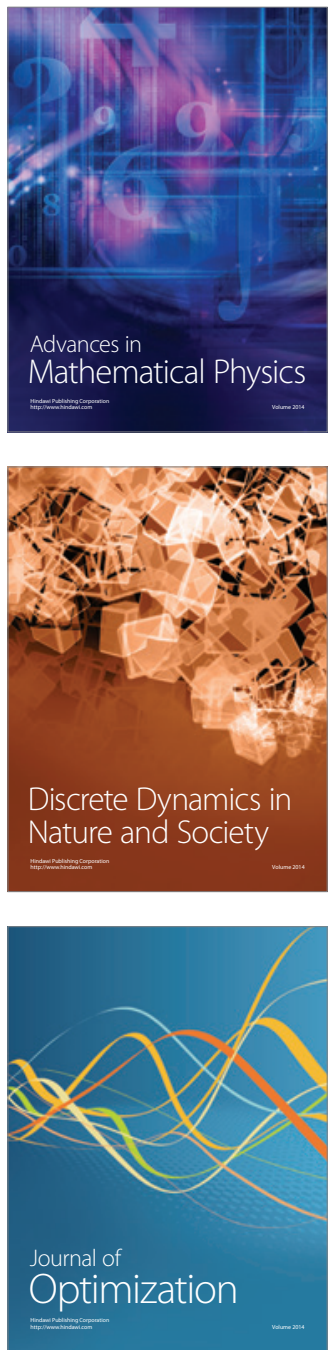